\title{
DEPPDB v.3: a portal to study electrostatic and other physical properties of genome DNA and its elements
}

\author{
A. Osypov ${ }^{1,2 *}$, G. Krutinin ${ }^{3}$, E. Krutinina 3 , P. Beskaravayny ${ }^{3}$, S. Kamzolova ${ }^{3}$ \\ ${ }^{1}$ Institute of Higher Nervous Activity and Neurophysiology RAS, Moscow, Russia \\ ${ }^{2}$ Institute of Theoretical and Experimental Biophysics RAS, Pushchino MR, Russia \\ ${ }^{3}$ Institute of Cell Biophysics RAS, Pushchino MR, Russia \\ *e-mail:aosypov@gmail.com
}

Key words: DNA electrostatics, transcription regulation, genome evolution

\begin{abstract}
Motivation and Aim: DNA is a highly charged molecule and its electrostatic and other physical properties define its shape in the functional space and greatly influence its interactions with different proteins, especially regulating transcription, in particular RNA-polymerases and transcription factors.

Methods and Algorithms: The electrostatic potential around the double-helical DNA molecule was calculated by the original method [1] using a specially developed program package [2,3]. Calculations of other physical properties are based on the di- and trinucleotide content. Different cross-correlation analysis algorithms are applied.
\end{abstract}

Results: DEPPDB contains all completely sequenced archaeal, bacterial, viral, mitochondrial, plastids and eukaryotic genomes according to current release of NCBI RefSeq [9]. Data for promoters, regulation sites, binding proteins etc. are incorporated from different databases and compiled from original literature annotation. All data are fully integrated, several tools are provided to support different forms of analysis. Calculation on the fly of the user-provided sequences and selected GeneBank records is available. DEPPDB can be considered as a portal or collection of databases on the electrostatic and other physical properties of whole genomes and different genome elements in different taxa and organisms: Promoter DB, Regulatory Sites (Transcription Factors, TF) DB, Gene Starts DB, Terminator DB, etc. as well as comprehensive analysis toolbox.Several calculation algorithms, data processing approaches and database architecture solutions were fundamentally revisited and fine tuned since the previous database releases to withstand the considerably increased data abundance.

Conclusion: DEPPDB was developed to hold and provide all available information on these properties of genome DNA combined with its sequence and annotation of biological and structural properties of genome elements and whole genomes, organized on a taxonomical basis.DB is available at http://deppdb.psn.ru.

Acknowledgements: Supported by the RFBR (No. 16-04-01865 and 18-34-00942).

References

1. Polozov R.V. et al. (1999) Electrostatic potentials of DNA. Comparative analysis of promoter and nonpromoter nucleotide sequences, J. Biomol. Struct. Dyn. 16(6):1135-1143.

2. Osypov A.A. et al. (2010) DEPPDB - DNA Electrostatic Potential Properties Database. Electrostatic Properties of Genome DNA, JBCB. 8(3):413-25.

3. Osypov A.A. et al. (2012) DEPPDB - DNA Electrostatic Potential Properties Database. Electrostatic Properties of Genome DNA elements, JBCB. 10(2):1241004. 